\title{
Apical Root Resorption: A Prospective Radiographic Study of Maxillary Incisors
}

\author{
Ilken Kocaderelia \\ Turkan Nadire Yesil ${ }^{b}$ \\ Pınar Sahin Veskec \\ Serdar Uysal ${ }^{d}$
}

\section{ABSTRACT}

Objectives: The aim of this study was to determine the prevalence of apical root resorption in maxillary incisors during the initial stages of active orthodontic treatment and to test the hypothesis that root resorption increases with the progress of the treatment.

Methods: The study sample consisted of 80 teeth of 20 patients ( 14 female, 6 male) with a mean age of $14.9 \pm 2.8$ years. Root resorption was determined with standardized digitized periapical radiographs. All the periapical radiographs were obtained at the beginning of orthodontic treatment (TO) and 3 months (T1), 6 months (T2) and 9 months (T3) after the beginning of the treatment by a paralleling device. They were digitalized as $600 \mathrm{dpi}$ with a flatbed scanner and analyzed by software for image analysis at 400x magnification utilizing a personal computer.

Results: All of the 4 maxillary incisors had an increasing amount of resorption during the 9-month period. The amount of root resorption between the time intervals was statistically significant $(P<.05)$.

Conclusions: Root resorption of maxillary incisors can be detected in the early stages of orthodontic treatment and appears to be related to treatment duration. According to 9-month evaluation period, apical root resorption is of limited clinical significance for the average orthodontic patient. (Eur J Dent 2011;5:318-323)

Key words: Orthodontics; Root resorption; Maxillary incisors; Prevalence.

a Professor and Head, Department of Orthodontics, Faculty of Dentistry, Hacettepe University, Ankara, Turkiye.

b Orthodontist, Center of Oral and Teeth Health, Amasya, Turkiye.

Research Assistant, Department of Orthodontics, Faculty of Dentistry, Hacettepe University, Ankara, Turkiye.

d Resident, Department of Oral Diagnosis and Radiology, Faculty of Dentistry, Hacettepe University, Ankara, Turkiye.

- Corresponding author: Itken Kocadereli Süslü Sokak No: 4/6, Tandoğan, Mebusevleri 06580, Ankara, Turkiye.

Phone : +90312 $2237137,+903123116461$

E-mail: ikocadereliahotmail.com

\section{INTRODUCTION}

Many clinical orthodontists has observed apical root resorption at the end of treatment and wanted to know what caused it. The application of external forces to the teeth to produce orthodontic tooth movement carries some calculated risks. One of these is irreversible root resorption. External apical root resorption is an undesirable sequel of orthodontic treatment that results in permanent loss of tooth structure from the root apex. Different types of orthodontic tooth movement may produce different mechanical stresses at varying locations with the root. $^{1}$ 
An extensive review concerning root resorption by Brezniak and Wasserstein ${ }^{2,3}$ indicated that multiple factors are involved in the mechanism. These include such matters as genetics and systemic factors, sex differences, type of tooth movement, magnitude of orthodontic force, duration and type of force. ${ }^{2,3}$ Some studies ${ }^{4,5}$ have aimed to elucidate the causal relationship between force application, tooth movement and root resorption by using scanning electron microscopy (SEM) and concluded that root resorption is time- and force-dependent, and the type of tooth movement also seems to play a role. All human teeth develop resorption lacunae on the pressure side of the root surfaces shortly after application of orthodontic forces ${ }^{1,6-8}$ starting at or near the periphery of hyalinized areas. ${ }^{1,6}$ Owman et $\mathrm{al}^{7}$ reported that the initial resorption lacunae are small and can be identified only by histological methods and orthodontically-induced root resorptions after 7 weeks of treatment, are verified histologically, and are not visible in periapical radiographs.

Radiographic examination of orthodontically treated patients showed some loss of root length. ${ }^{9-11}$ The maxillary incisors have been regarded as the most susceptible to root resorption, particularly those with blunt or pipette-shaped roots. ${ }^{9,12-13}$ A 3-month radiographic control has been recommended for maxillary incisors with an enhanced risk of root resorption. ${ }^{14}$ There are different opinions regarding whether a correlation exists between the duration of active treatment and the incidence and degree of root resorption. ${ }^{14-18}$

The aims of this study were to determine the prevalence of apical root resorption in maxillary central and lateral incisors during the initial stages of active orthodontic treatment and to test the hypothesis that root resorption increases with the progress of the treatment.

\section{MATERIALS AND METHODS}

The study sample consisted of 20 Turkish Anatolian patients ( 14 females and 6 males), with a mean age of $14.9 \pm 2.8$ years (range 11.6 to 22.3 years). After ethical clearance from Ankara Clinic Researches Ethical Committee No: 3 with record number: HEK 09/243, the patients who were referred to Department of Orthodontics for the treatment of their malocclusion were selected randomly. The initial malocclusion of the patients was Angle Class I with anterior crowding. The severity of the crowding $(4-6 \mathrm{~mm})$ was similar among the patients. All the patients were treated with multibonded pre-adjusted appliances (Roth brackets) with .018" bracket slots. During the treatment edgewise mechanics were used. The initial arch wire was .016" Nickel-Titanium, then .016x.016" $\mathrm{Ni}-\mathrm{Ti}, .016 x .022 " \mathrm{Ni}-\mathrm{Ti}$ arch wires were used respectively. The same clinician (TNY) treated all the patients. The total sum of 80 teeth, the maxillary right and left, and central and lateral incisors (12, $11,21,22)$ of the 20 patients were observed radiographically for a period of 9 months. The exclusion criteria of the patients were: history of previous orthodontic or endodontic treatment, traumatic injuries, crown or root fractures, severely dilacerated roots, congenitally missing laterals, incomplete root formation at the start of treatment, and maxillary incisors with caries.

All the periapical radiographs were obtained before the orthodontic treatment (TO), 3 (T1), 6 (T2) and 9 (T3) months after the beginning of the treatment by a paralleling device (Dentsply Rinn, Rinn Cooperation, Elgin, IL, USA), and were digitalized as 8 bit, $600 \mathrm{dpi}$ with a flatbed scanner (EPSON EXPRESSION 10000 XL, Seiko Epson Co., Nagano, Japan) and analyzed in a software for image analysis (ImageJ 1.410, NIH, Maryland, MD, USA) at 400x magnification in a personal computer.

Mesial (a) and distal (b) incisal edge points, mesial(c) and distal (d) cementoenamel junction (CEJ) points, and mesial (e) and distal (f) edge points of the apical foramen were identified on pre-treatment and post-treatment periapical radiographs. The two points were connected by a line drawn from mesial point to distal point at the incisal, cementoenamel junction and apical levels (Figure 1). Midpoints of the drawn horizontal lines ( $a-b$ and e-f) were connected vertically. The vertical line connecting the two horizontal lines between mesial and distal edge points (a-b), and the cement enamel junction ( $c-d$ ) is called "crown height" (A). The vertical line connecting the two horizontal lines defined between the cementoenamel junction ( $c-d)$ and the apical foramen (e-f) is called as "root length" (B). The sum of "crown height" and "root length" gives the "total tooth length." In this study, to measure the amount of apical root resorption, "root length" was used.

To correct differences in projection, a defined methodology was used ${ }^{19}$ and the roots were adjusted in the following manner. The averages of the pre-treatment and post-treatment crown lengths were computed by the following formula:

$\mathrm{Cx}=(\mathrm{C} 1+\mathrm{C} 2) / 2$

(the average represented as $\mathrm{Cx}$, pretreatment 
crown length represented as $\mathrm{C} 1$ and post-treatment crown length represented as $\mathrm{C} 2$ ).

Then, the pre-treatment and post-treatment root lengths were adjusted to the averaged crown lengths. The following formula was used to adjust the root lengths:

$\mathrm{R} 1$ (adjusted) $=\mathrm{R} 1 \times(\mathrm{C} \times / \mathrm{C} 1)$.

The amount of root resorption was calculated as the difference between the second (post-treatment) and first (pre-treatment) root length measurements. No reference brackets failed during the observation period.

\section{Statistical analysis}

Statistical analysis was carried out with SPSS for Windows version 15.0 statistical software (SPSS Inc., Chicago, IL, USA). Variables were presented as mean \pm standard deviation. Repeated measures ANOVA were used to determine the

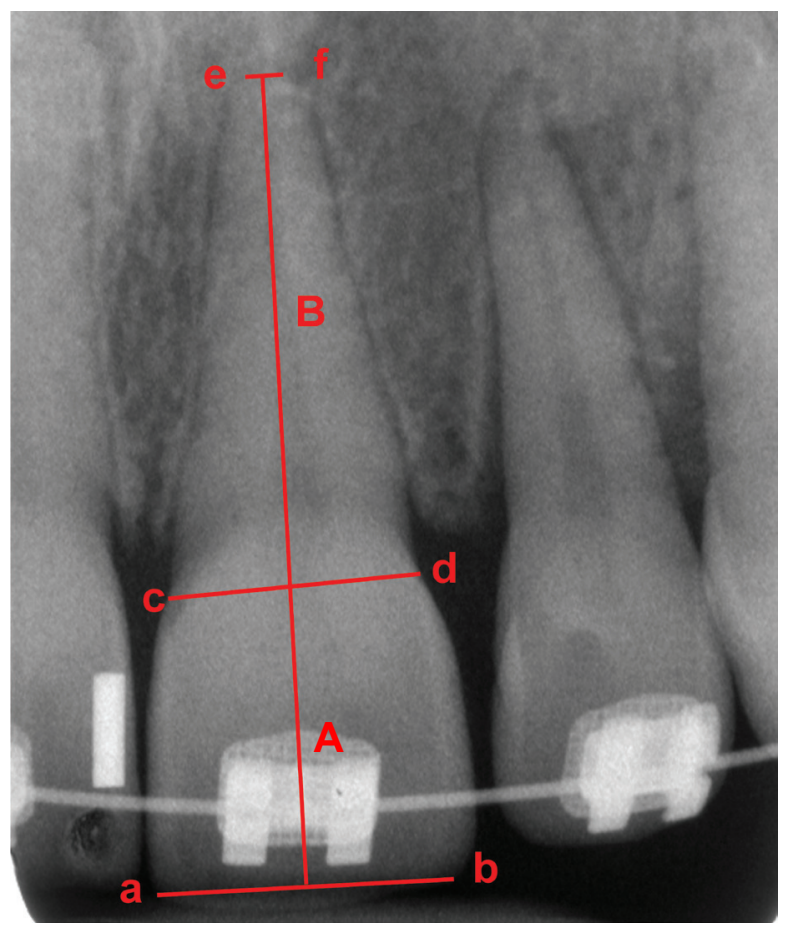

Figure 1 a: Mesial incisal edge point, b: distal incisal edge point, c: mesial cementoenamel junction point, d: distal cementoenamel junction point, e: mesial edge point of the apical foramen, $f$ : distal edge point of the apical foramen, $A$ : crown height, $B$ : root length, $C(A+B)$ : total tooth length. amount of root resorption difference between time intervals. Multiple comparisons were performed with the Bonferroni test. Statistical significance rate was stated at the 0.05 level.

The measurement error was analyzed by intraclass correlation coefficient test and radiographs of the tooth (12) were taken at T0, T1, T2 and T3 time periods and were re-measured using the same methodology. Before orthodontic treatment and 6 th month were find $99 \%(P<.001), 3^{\text {rd }}$ and $9^{\text {th }}$ month $100 \%(P<.001)$.

\section{RESULTS}

The statistical evaluation of apical root resorption showed the mean rate of apical root resorption was constant during the three months periods for all four maxillary incisors (Table 1). When comparing T0 to T1, the amount of resorption was $0.16 \pm 0.21 \mathrm{~mm}, 0.24 \pm 0.23 \mathrm{~mm}$, and $0.2 \pm 0.18 \mathrm{~mm}$ for teeth 11, 21 and 22, respectively, and were statistically significant $(\mathrm{P}<.05)($ Table 2$)$. For tooth 12 , the amount of resorption was $0.34 \pm 0.55 \mathrm{~mm}$ which was not statistically significant ( $P>.05)$ (Table 2).

When comparing T0-T2, TO-T3, T1-T2 and T1T3 periods, the amount of resorption was statistically significant for all the maxillary incisors $(P<.05)$ (Table 2).

When comparing the T2-T3 period, the amounts of resorption for teeth 11,12 , and 21 were statistically significant with $0.24 \pm 0.26 \mathrm{~mm}, 0.3 \pm 0.35 \mathrm{~mm}$, and $0.16 \pm 0.12 \mathrm{~mm}$, respectively. For tooth 22 , the amount of resorption was not statistically significant with $0.07 \pm 0.25 \mathrm{~mm}(P>.05)$ (Table 2$)$. At the end of 9 months, tooth 12 had the maximum amount of resorption with $0.98 \pm 0.74 \mathrm{~mm}$; and tooth $22 \mathrm{had}$ the minimum amount of resorption with $0.6 \pm 0.39$ $\mathrm{mm}$ (Figure 2).

\section{DISCUSSION}

External apical root resorption (EARR) can be defined as blunting or shortening of the root apex, a condition often associated with orthodontic treatment. ${ }^{20}$ The teeth that are more susceptible to

Table 1. Mean root lengths of maxillary incisors during periods.

\begin{tabular}{|c|c|c|c|c|c|c|c|c|c|}
\hline \multirow[b]{2}{*}{ Teeth } & \multicolumn{2}{|l|}{ TO } & \multicolumn{2}{|l|}{ T1 } & \multicolumn{2}{|l|}{ T2 } & \multicolumn{2}{|l|}{ T3 } & \multirow[b]{2}{*}{$\mathrm{P}$} \\
\hline & Mean $(\mathrm{mm})$ & SD & Mean $(\mathrm{mm})$ & SD & Mean $(\mathrm{mm})$ & SD & Mean $(\mathrm{mm})$ & SD & \\
\hline 11 & 17.07 & 1.9 & 16.9 & 1.8 & 16.5 & 1.8 & 16.2 & 1.9 & $* * *$ \\
\hline 12 & 15.5 & 1.8 & 15.1 & 1.7 & 14.8 & 1.9 & 14.5 & 1.8 & $* * *$ \\
\hline 21 & 17.1 & 1.9 & 16.8 & 2 & 16.5 & 2 & 16.3 & 2 & $* * *$ \\
\hline 22 & 15.3 & 1.6 & 15.1 & 1.7 & 14.7 & 1.9 & 14.7 & 1.7 & $* * *$ \\
\hline
\end{tabular}

***: $P<.001$ 
EARR are the maxillary and mandibular incisors, especially the maxillary lateral incisors..$^{15,21-23}$ Previous studies ${ }^{10,23-25}$ have shown a relation between narrow roots and resorption. As such root forms are common in the maxillary lateral rather than in

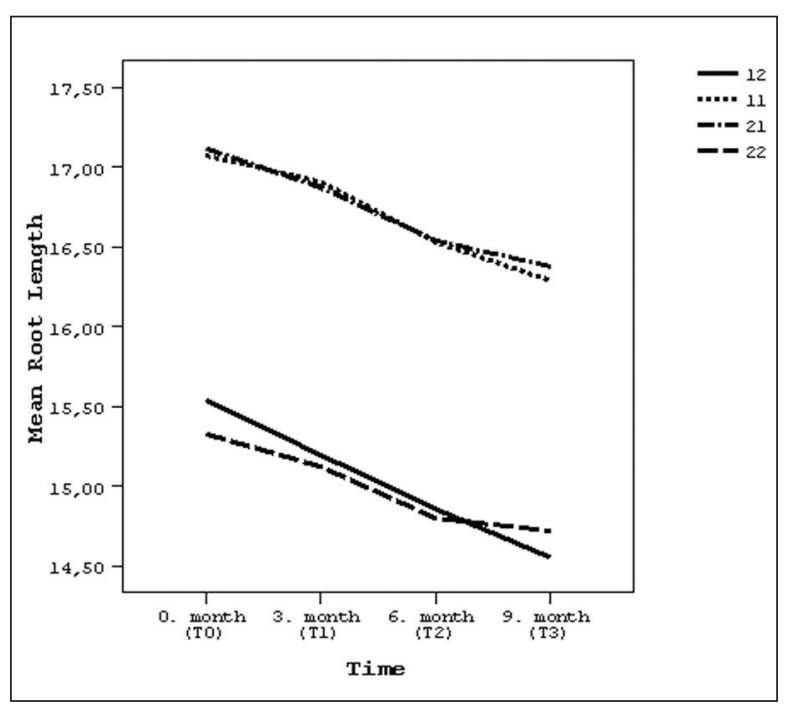

Figure 2. The mean root lengths of upper incisors during 9 months. the maxillary central incisors, the common finding is that maxillary lateral incisors experience resorption more than other teeth during orthodontic treatment ${ }^{11,15,22}$ may not be unexpected.

In this study we tried to investigate the amount of EARR on maxillary central and lateral incisors of patients treated with the conventional bracket system during the initial stages of active orthodontic treatment and to answer the question whether root resorption increases with the progress of the orthodontic treatment. Studies based on random measurements of tooth lengths on standardized periapical radiographs made before and after active orthodontic treatment conclude that the sample mean of the averaged amount of resorption of all six maxillary anterior teeth, ${ }^{22}$ of the four maxillary incisors, ${ }^{15,21}$ or each pair of maxillary central and lateral incisors ${ }^{15,21-23}$ is less than $1.5 \mathrm{~mm}$. In keeping with these studies, we found the greatest resorption in tooth 12, with the amount of $1.47 \mathrm{~mm}$ in one patient (Figure 3). Accordingly, apical root resorption is of limited clinical significance for the

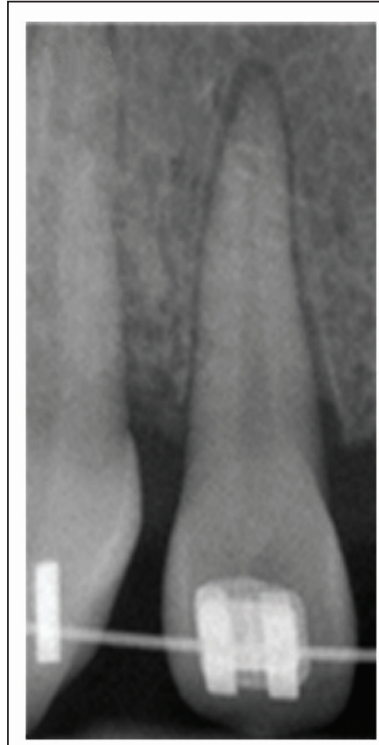

T 0

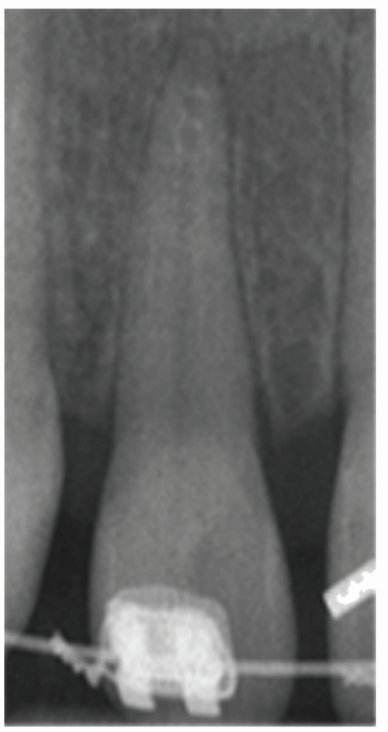

T 1

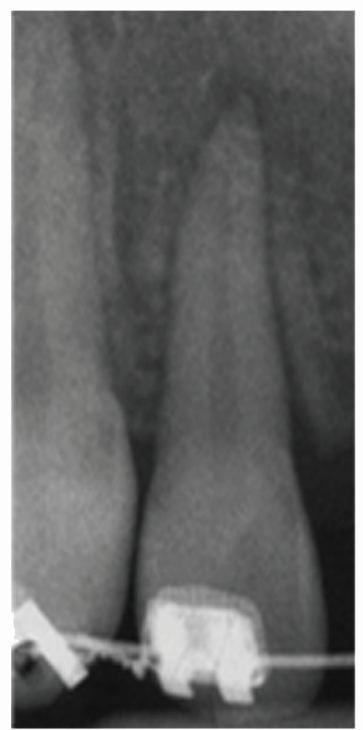

T 2

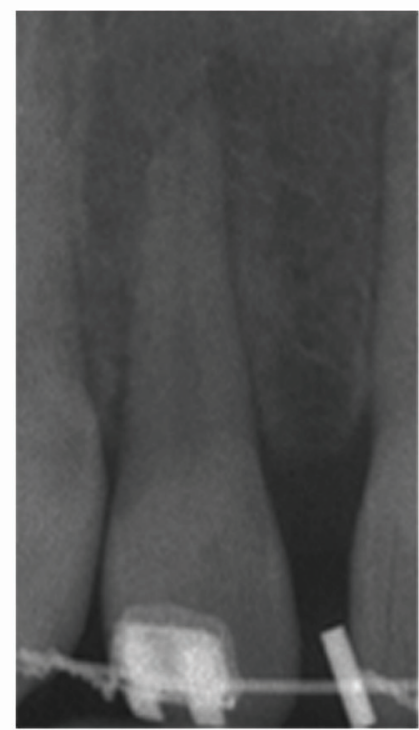

T 3

Figure 3. Periapical radiographs of the patient showing the greatest resorption in tooth 12 during TO-T3.

Table 2. The amount of root resorption between periods.

\begin{tabular}{|c|c|c|c|c|c|c|c|c|c|c|c|c|c|c|c|c|c|c|}
\hline \multirow[b]{2}{*}{ Teeth } & \multicolumn{3}{|c|}{ T0-T1 } & \multicolumn{3}{|c|}{ T0-T2 } & \multicolumn{3}{|c|}{ T0-T3 } & \multicolumn{3}{|c|}{ T1-T2 } & \multicolumn{3}{|c|}{ T1-T3 } & \multicolumn{3}{|c|}{ T2-T3 } \\
\hline & $\begin{array}{l}\text { Mean } \\
(\mathrm{mm})\end{array}$ & SD & $P$ & $\begin{array}{l}\text { Mean } \\
(\mathrm{mm})\end{array}$ & SD & $P$ & $\begin{array}{l}\text { Mean } \\
\text { (mm) }\end{array}$ & $\mathrm{SD}$ & $P$ & $\begin{array}{l}\text { Mean } \\
(\mathrm{mm})\end{array}$ & SD & $P$ & $\begin{array}{l}\text { Mean } \\
(\mathrm{mm})\end{array}$ & SD & $P$ & $\begin{array}{l}\text { Mean } \\
(\mathrm{mm})\end{array}$ & SD & $P$ \\
\hline 11 & 0.16 & 0.21 & $*$ & 0.54 & 0.47 & $*$ & 0.78 & 0.62 & $*$ & 0.37 & 0.4 & $* *$ & 0.62 & 0.5 & $*$ & 0.24 & 0.3 & $* *$ \\
\hline 12 & 0.34 & 0.55 & ns & 0.68 & 0.75 & $* *$ & 0.98 & 0.74 & $*$ & 0.33 & 0.5 & $*$ & 0.64 & 0.4 & $*$ & 0.3 & 0.4 & $* *$ \\
\hline 21 & 0.24 & 0.23 & $* * *$ & 0.57 & 0.38 & $*$ & 0.73 & 0.4 & $*$ & 0.33 & 0.3 & $* * *$ & 0.49 & 0.3 & $*$ & 0.16 & 0.1 & $*$ \\
\hline 22 & 0.2 & 0.18 & $* * *$ & 0.52 & 0.48 & $* * *$ & 0.6 & 0.39 & $*$ & 0.32 & 0.4 & $*$ & 0.4 & 0.3 & $*$ & 0.07 & 0.3 & ns \\
\hline
\end{tabular}

ns: not significant; *: $\mathrm{P}<.05 ;{ }^{* *}$ : $\mathrm{P}<.01$; ***: $\mathrm{P}<.001$ 
average patient. Available clinical studies on apical orthodontic root resorption are almost invariably designed in retrospect relative to the timing of active treatment, selecting pretreatment and post-treatment records to be measured from various patient files. Results about prevalence, severity, and morphologic risk factors are not likely to be different in studies designed before treatment started, if patient materials are representative, radiographs are of sufficient quality, and appropriate records are available for collecting relevant morphologic parameters. In both situations, the experimental designs are identical, analyzing data collected according to a predetermined protocol after all active treatment. Information from representative patient samples, based on random measurements of tooth lengths on pretreatment and post-treatment radiographs made according to a standardized paralleling technique, are, therefore, likely to be valid. Also, extraoral x-rays are considered less accurate than periapical radiographs in studying the extent of EARR. ${ }^{26}$ Sameshima and Asgarifar ${ }^{27}$ showed that the amount of EARR might be overestimated up to $20 \%$ on panoramic films.

In this study, periapical radiographs that were obtained according to a standardized paralleling technique at predetermined stages of treatment were used.

In this study, gender and age of the patients were not considered because a number of studies have evaluated the role of age and gender ${ }^{10,11,23,26,28}$ of the patients and found no correlation.

Mirabella et $\mathrm{al}^{24}$ and Baumrind ${ }^{29}$ reported no association between type of initial malocclusion, treatment time, use of rectangular arch wires, and root resorption. Adversely, many recent studies $^{10,11,26,30}$ have found that longer treatment time was significantly associated with increased root resorption especially for maxillary incisors.

Previous studies also generally support the significance of extended treatment time as the cause of root resorption. 13,25,29,31,32 In accordance with these studies, the results of our study confirmed that root resorption increases by the progress of the treatment. Table 1 shows increasing amounts of root resorption during the 9-month period.

The low explained variance of identified risk factors suggests the major reason for the variation in orthodontic root resorption could be individual predisposition. If so, predisposed patients might experience root resorption already in the initial leveling stages. Very few studies explored that hypothesis. ${ }^{13,14}$ Levander ${ }^{13}$ evaluated 390 teeth in 98 patients. However availability of only nonstandardized periapical radiographs necessitated simultaneous, subjective scoring of the pretreatment and post-treatment radiographs, introducing a risk of bias. Also, the crude scoring scale might not have been sufficiently accurate to depict the small changes likely to occur during the initial treatment stages. In this study, total tooth length, root length and crown height were measured from the beginning of treatment with the periods of 3 months until 9 months and all 4 maxillary incisors showed root resorption at the first period (TO-T1) and, except for tooth 12, the amount of root resorption of maxillary incisors was statistically significant $(P<.05)(T a b l e ~ 2)$. This finding is in accordance with Levander et $\mathrm{al}^{33}$ and Artun et al. ${ }^{10}$ However, Levander et al ${ }^{33}$ evaluated maxillary incisors at 3 and 6 months, while we investigated for 3 more months ( 9 months) to state that EARR increases with time. Also, in our study, all radiographs were taken more frequently than in the study by Artun et al. ${ }^{10}$ The weakness of our study was the sample size.

\section{CONCLUSIONS}

- External apical root resorption (EARR) can be detected in the early stages of orthodontic treatment.

- EARR appears to be related to orthodontic forces from the beginning of the treatment.

- According to the 9-month evaluation period, apical root resorption is of limited clinical significance.

\section{REFERENCES}

1. Reitan K. Initial tissue behavior during apical root resorption. Angle Orthod 1974;44:68-82.

2. Brezniak N, Wasserstein A. Root resorption after orthodontic treatment: Part 1. Literature review. Am J Orthod Dentofacial Orthop 1993;103:62-66.

3. Brezniak N, Wasserstein A. Root resorption after orthodontic treatment: Part 2. Literature review. Am J Orthod Dentofacial Orthop 1993;103:138-146.

4. Casa MA, Faltin RM, Faltin K, Sander FG, Arana-Chavez VE. Root resorptions in upper first premolars after application of continuous torque moment. Intra-individual study. J Orofac Orthop 2001;62:285-295.

5. Faltin RM, Arana-Chavez VE, Faltin K, Sander FG, Wichelhaus $A$. Root resorptions in upper first premolars after application of continuous intrusive forces. Intra-individual study. J Orofac Orthop 1998;59:208-219. 
6. Kvam E. Scanning electron microscopy of human premolars following experimental tooth movement. Trans Eur Orthod Soc 1972:381-391.

7. Owman-Moll P, Kurol J, Lundgren D. Repair of orthodontically induced root resorption in adolescents. Angle Orthod 1995;65:403-408.

8. Kurol J, Owman-Moll P, Lundgren D. Time-related root resorption after application of a controlled continuous orthodontic force. Am J Orthod Dentofacial Orthop 1996;110:303310.

9. Remington DN, Joondeph DR, Årtun J, Riedel RA, Chapko MK. Long-term evaluation of root resorption occurring during orthodontic treatment. Am J Orthod Dentofacial Orthop 1989;96:43-46.

10. Artun J, Smale I, Behbehani F, Doppel D, Van't Hof M, Kuijperts-Jagtman AM. Apical root resorption six and 12 months after initiation of fixed orthodontic appliance therapy. Angle Orthod 2005;75:919-926.

11. Smale I, Årtun J, Behbehani F, Doppel D, van't Hof M, Kuijpers-Jagtman AM. Apical root resorption 6 months after initiation of fixed orthodontic appliance therapy. Am J Orthod Dentofacial Orthop 2005;128:57-67.

12. Newman WG. Possible etiologic factors in external root resorption. Am J Orthod 1975;67:539-552.

13. Levander E, Malmgren O. Evaluation of the risk of root resorption during orthodontic treatment: a study of upper incisors. Eur J Orthod 1988;10:30-38.

14. Levander E, Malgren O, Stenback K: Apical root resorption during orthodontic treatment of patients with multiple aplasia: a study of maxillary incisors. Eur $J$ Orthod 1998;20:427-434.

15. Linge $L$, Linge BO. Patient characteristics and treatment variables associated with apical root resorption during orthodontic treatment. Am J Orthod Dentofacial Orthop 1991;99:35-43.

16. Beck BW, Haris EF. Apical root resorption in orthodontically treated subjects: analysis of edgewise and light wire mechanics, Am J Orthod Dentofacial Orthop 1994;105:350361.

17. Mavragani M, Vergari A, Selliseth NJ, Boe OE, Wisth PJ. A radiographic comparison of apical root resorption after orthodontic treatment with a Standard edgewise and a straight-wire edgewise technique. Eur J Orthod 2000;22:665-674.

18. Brin I, Tulloch JFC, Koroluk L, Philips C. External apical root resorption in Class II malocclusion: a retrospective review of 1-versus 2-phase treatment. Am J Orthod Dentofacial Orthop 2003;124:151-156.
19. McFadden WM, Engstrom C, Engstrom H, Anholm M. A study of the relationship between incisor intrusion and root shortening. Am J Orthod Dentofacial Orthop 1989;96:390396.

20. Malmgren O, Levander E. Minimizing orthodontically induced root resorption. In: Graber T, Eliades T, Athanasiou $A E$, editors. Risk management in orthodontics: experts' guide to malpractice. Chicago: Quintessence; 2004. p. 6175.

21. Linge BO, Linge L. Apical root resorption in upper and anterior teeth. Eur J Orthod 1983;5:173-183.

22. Mirabella AD, Årtun J. Prevalence and severity of apical root resorption in upper anterior teeth in adult orthodontic patients. Eur J Orthod 1995;17:93-99.

23. Sameshima GT, Sinclair PM. Predicting and preventing root resorption: part I. Diagnostic factors. Am J Orthod Dentofacial Orthop 2001;119:505-510.

24. Mirabella AD, Årtun J. Risk factors for apical root resorption of maxillary anterior teeth in adult orthodontic patients. Am J Orthod Dentofacialial Orthop 1995;108:48-55.

25. Taithongchai R, Sookorn K, Killany DM. Facial and dentoalveolar structures and the prediction of apical root shortening. Am J Orthod Dentofacial Orthop 1996;110:296-302.

26. Pandis N, Nasika M, Polychronopoulou A, Eliades T. External apical root resorption in patients treated with conventional and self-ligating brackets. Am J Orthod Dentofacial Orthop 2008;134:646-651.

27. Sameshima GT, Asgarifar KO. Assessment of root resorption and root shape: periapical vs panoramic films. Angle Orthod 2001;71:185-189.

28. Harris EF, Baker WC. Loss of root length and crestal bone height before and during treatment in adolescent and adult orthodontic patients. Am J Orthod Dentofacial Orthop 1990;98:463-469.

29. Baumrind S, Korn EL, Boyd RL. Apical root resorption in orthodontically treated adults. Am J Orthod Dentofacial Orthop 1996;110:311-320.

30. Sameshima GT, Sinclair PM. Predicting and preventing root resorption: part II. Treatment factors. Am J Orthod Dentofacial Orthop 2001;119:511-515.

31. Vlaskalic V, Boyd RL, Baumrind S. Etiology and sequelae of root resorption. Semin Orthod 1998;4:124-131.

32. Hendrix I, Carels C, Kuijpers-Jagtman AM, Van'T Hof M. A radiographic study of posterior apical root resorption in orthodontic patients. Am J Orthod Dentofacial Orthop 1994; 105:345-349.

33. Levander E, Malmgren 0. Early radiographic diagnosis of apical root resorption during orthodontic treatment: a study of maxillary incisors. Eur J Orthod 1998;20:57-63. 